\title{
More on the Mathematics of the DLF Theory: Embedding of the Oscillator World L into Segal's Compact Cosmos D
}

\author{
J. Y. Feng ${ }^{*}$ and A. V. Levichev* \\ Department of Mathematics \\ Boston University \\ Boston, Massachusetts 02215 USA
}

Received: December 16, 2012

Accepted: April 2, 2013

\section{ABSTRACT}

The DLF theory can be understood as an attempt to modify the Standard Model by flexing the Poincare symmetry to certain 7-dimensional symmetries. The D part of the theory is known as Segal's Chronometry which is based on compact cosmos $D=U(2)$ with the $S U(2,2)$ fractional linear action on it. The oscillator group is viewed as a subgroup $L_{G}$ of the conformal group $G=S U(2,2)$ and certain $L_{G}$-orbits $L$ in $D$ are studied. We prove existence of such $L$ and of such an embedding of $F=U(1,1)$ into $D$, that $D$ differs from $F$ by a certain torus whereas $D$ differs from $L$ by a circle on that torus. In the general $U(p, q)$ vs $U(p+q)$ case, the Sviderskiy formula is described - as a tribute to the late Oleg S. Sviderskiy (July 311969 - March 30 2011).

\section{INTRODUCTION}

The DLF's overall goal is to develop a comprehensive mathematical model which is an extension of the current Standard Model of particles and interactions. We note that some expected experimental predictions (within the Standard Model) have remained unobserved, the most recent example of which is the lack of detection of the Higgs boson in current Large Hadron Collider experiments. If the new approach is successfully vetted in the context of experimental physics, it would modify certain views of physical laws, and possibly influence some other disciplines, similarly to transitions in viewpoint originally brought on by special relativity. In the context of Segal's chronometric theory it has been shown [1] that exact invariance under a 7dimensional isometry group $K$ of the Einstein static universe (the latter being the universal cover of the chronometric compact cosmos $D)$ guarantees approximate Poincare invariance and that it is far beyond the accuracy of currently available devices to experimentally decide between the two types of invariance. Having this in mind, the DLF theory (see [2]) can be viewed as an attempt to modify

*Emails: jfeng8@bu.edu; levit@math.bu.edu the Standard Model by flexing the Poincare symmetry to three types of 7dimensional symmetries (one of them being the $K$-symmetry).

In terms of the DLF terminology, the current article is primarily dedicated to the L-ingredient of the theory. The oscillator group will be introduced below as a subgroup $L_{G}$ of the conformal group $G=S U(2,2)$, whereas by $L$ we will denote a certain $L_{G}$-orbit in $D$. The main inquiries and statements of our article are motivated by the (still pending) necessity to deal with L-based parallelizations (additionally to parallelizations of vector bundles introduced in [3]: see the relevant discussion in [2] (Section 7). In the DLF context, the oscillator Lie algebra / has been introduced in Section 3.3 of [2]. Our Section 2 introduces both $/$ and (some of the) corresponding Lie groups.

Part of the content of Section 3 is a tribute to the late Oleg S. Sviderskiy (July 311969 - March 30 2011). Overall, the current article provides mathematical justification to some of the claims of [2].

\section{REALIZATIONS OF THE OSCILLATOR GROUP AS SUBGROUPS IN U(2,1) AND $\mathrm{SU}(2,2)$}

Following [4], we now introduce the oscillator Lie algebra as the totality of all matrices 


$$
\left[\begin{array}{ccc}
i x_{1} & z & -i x_{1} \\
-\bar{z} & i x_{4} & \bar{z} \\
i x_{1} & z & -i x_{1}
\end{array}\right]
$$

where $z=x_{2}+i x_{3}$. Here (and below) variables $x_{1}, x_{2}, x_{3}, x_{4}$ are real. Introduce Lie algebra $u(2,1)$ as the totality of all 3 by 3 matrices $m$ (complex entries allowed) which satisfy

$$
m s+s m^{*}=0
$$

where $s$ a diagonal matrix with entries 1,1 , -1 .

Proposition 1. Each matrix (1) belongs to $u(2,1)$.

The straightforward proof is omitted.

Remark 1. Each matrix (1) can be viewed as a linear combination of $e_{1}, e_{2}, e_{3}, e_{4}$. Namely,

$$
\begin{array}{rlr}
e_{1}=\left[\begin{array}{ccc}
i & 0 & -i \\
0 & 0 & 0 \\
i & 0 & -i
\end{array}\right], & e_{2}=\left[\begin{array}{ccc}
0 & 1 & 0 \\
-1 & 0 & 1 \\
0 & 1 & 0
\end{array}\right], \\
e_{3}=\left[\begin{array}{ccc}
0 & i & 0 \\
i & 0 & -i \\
0 & i & 0
\end{array}\right], & e_{4}=\left[\begin{array}{lll}
0 & 0 & 0 \\
0 & i & 0 \\
0 & 0 & 0
\end{array}\right] .
\end{array}
$$

The commutation relations are as follows:

$$
\begin{gathered}
{\left[e_{2}, e_{4}\right]=e_{3},} \\
{\left[e_{4}, e_{3}\right]=e_{2},} \\
{\left[e_{2}, e_{3}\right]=2 e_{1} .}
\end{gathered}
$$

The last relation differs by factor 2 from the one in Section 3.3 of [2]. The two Lie algebras are isomorphic. The following notation is used below: $z=x_{2}+i x_{3}, e=e^{i x_{4}}$, $m=z \bar{z} / 2$.

Theorem 1. The totality of all matrices

$$
U=\left[\begin{array}{ccc}
1-m+i x_{1} & z & m-i x_{1} \\
-e \bar{z} & e & e \bar{z} \\
-m+i x_{1} & z & 1+m-i x_{1}
\end{array}\right]
$$

is a (closed) oscillator subgroup in $U(2,1)$.

Proof (outline). The matrix (3) is the product of $\exp \left(x_{4} \boldsymbol{e}_{4}\right)$ and $\exp n$ (in that order) where $n$ is the linear combination of $e_{1}, e_{2}, e_{3}$ from above. This observation guarantees, already, that the totality of all matrices (3) is a group. It is helpful however to determine precise expressions for the (3) parameters of the product $U \widetilde{U}$.They are not given here since we will only need these expressions in later study. Proposition 1 guarantees that it is a subgroup of $U(2,1)$.

Remark 2. It is clear from (3) that our oscillator subgroup has topology of $S^{1}$ times $R^{3}$. The (standard) group operation for its universal covering group (with the $R^{4}$ topology) can be found on p.411 of [5].

We now introduce oscillator Lie group as a subgroup $L_{G}$ in $G=S U(2,2)$. The latter group and its fractional linear action on $U(2)$

$$
g(Z)=(\mathrm{A} Z+\mathrm{B})(\mathrm{C} Z+\mathrm{D})^{-1}
$$

(where an element $g$ is determined by $2 \times 2$ blocks A, B, C, D) is defined in [2] (Section $6)$. Consider the set of all 4 by 4 matrices $g$ of the form

$$
g=U_{+} e^{-1}
$$

(direct sum of a matrix (3) with the 1 by 1 matrix $e^{-1}$ where $\left.e=e^{i x_{4}}\right)$.

Theorem 2. The totality $L_{G}$ of all matrices (4) is a closed oscillator subgroup in $G=S U(2,2)$. The $L_{G}$-orbit $L$ of the matrix -1 under fractional linear action is all $D=U(2)$ but a certain circle (described below). The stationary $L_{G}$-subgroup of $\mathbf{- 1}$ is the following set (of two elements): $z=0, x_{1}=0$, $\exp \left(2 i x_{4}\right)=1$

Proof. The determinant of the (3) matrix $U$ is e which is why the matrix $g(2.4)$ is in SU(2,2). From (4), we form the following matrices:

$$
A=\left[\begin{array}{cc}
1-m+i x_{1} & z \\
-e \bar{z} & e
\end{array}\right],
$$

$$
\begin{aligned}
& \mathrm{B}=\left[\begin{array}{cc}
m-i x_{1} & 0 \\
e \bar{z} & 0
\end{array}\right], \\
& \mathrm{C}=\left[\begin{array}{cc}
-m+i x_{1} & z \\
0 & 0
\end{array}\right],
\end{aligned}
$$

$$
\mathrm{D}=\left[\begin{array}{cc}
1+m-i x_{1} & 0 \\
0 & e^{-1}
\end{array}\right]
$$

Fractional linear action applied to an element $Z$ from $U(2)$ (we then take $Z=-1$ ) results in: 


$$
\begin{gathered}
(\mathrm{A} Z+\mathrm{B})(\mathrm{C} Z+\mathrm{D})^{-1} \\
=\left(\left[\begin{array}{cc}
1-m+i x_{1} & z \\
-e \bar{z} & e
\end{array}\right] Z+\left[\begin{array}{cc}
m-i x_{1} & 0 \\
e \bar{z} & 0
\end{array}\right]\right)\left(\left[\begin{array}{cc}
-m+i x_{1} & z \\
0 & 0
\end{array}\right] Z+\left[\begin{array}{cc}
1+m-i x_{1} & 0 \\
0 & e^{-1}
\end{array}\right]\right)^{-1} \\
=\left(\frac{1}{1+2 m-2 i x_{1}}\right)\left[\begin{array}{cc}
-1+2 m-2 i x_{1} & -z \\
2 e \bar{z} & -e
\end{array}\right]\left[\begin{array}{cc}
1 & e z \\
0 & e\left(1+2 m-2 i x_{1}\right)
\end{array}\right] \\
=v\left[\begin{array}{cc}
2 m-1-2 i x_{1} & -2 e z \\
2 e \bar{z} & e^{2}\left(2 m-1+2 i x_{1}\right)
\end{array}\right]
\end{gathered}
$$

In other words, an element $M$ of the orbit is

$$
\cup\left[\begin{array}{cc}
2 m-1-2 i x_{1} & -2 e z \\
2 e \bar{z} & e^{2}\left(2 m-1+2 i x_{1}\right)
\end{array}\right],
$$

where $\mathrm{v}=\left(\frac{1}{1+2 m-2 i x_{1}}\right)$.

It is well-known that an arbitrary $Z$ from $U(2)$ can be viewed as

$$
\left[\begin{array}{ll}
1 & 0 \\
0 & d
\end{array}\right]\left[\begin{array}{ll}
u_{4}+i u_{3} & u_{2}+i u_{1} \\
i u_{1}-u_{2} & u_{4}-i u_{3}
\end{array}\right]
$$

where $|d|=1$, and

$$
u_{1}{ }^{2}+u_{2}{ }^{2}+u_{3}{ }^{2}+u_{4}{ }^{2}=1,
$$

with real variables $u_{1}, u_{2}, u_{3}, u_{4}$. Then (viewing $Z$ as given) the equality $Z=M$ (of two matrices) reads:

$$
\begin{gathered}
{\left[\begin{array}{ll}
1 & 0 \\
0 & d
\end{array}\right]\left[\begin{array}{ll}
u_{4}+i u_{3} & u_{2}+i u_{1} \\
i u_{1}-u_{2} & u_{4}-i u_{3}
\end{array}\right]=} \\
\cup\left[\begin{array}{cc}
2 m-1-2 i x_{1} & -2 e z \\
2 e \bar{z} & e^{2}\left(2 m-1+2 i x_{1}\right)
\end{array}\right]
\end{gathered}
$$

Start with the equality of the first entries in the first rows:

$u_{4}+i u_{3}=\frac{2 m-1-2 i x_{1}}{1+2 m-2 i x_{1}}=\frac{4 m^{2}+4 x_{1}^{2}-1-4 i x_{1}}{(2 m+1)^{2}+4 x_{1}^{2}}$

The case $u_{4}=1$ (which implies $u_{1}=u_{2}=u_{3}$ $=0$ ) has no solution, we will return to it later. Otherwise, the above is equivalent to

$$
u_{4}=\frac{4 m^{2}+4 x_{1}^{2}-1}{(2 m+1)^{2}+4 x_{1}^{2}} \text { and } u_{3}=\frac{-4 x_{1}}{(2 m+1)^{2}+4 x_{1}^{2}} \text {. }
$$

This system is solved by

$$
x_{1}=\frac{-\boldsymbol{u}_{3}}{\left(1-u_{4}\right)^{2}+\boldsymbol{u}_{3}^{2}} \text { and } 2 m=\frac{1-\boldsymbol{u}_{3}{ }^{2}-\boldsymbol{u}_{4}{ }^{2}}{\left(1-\boldsymbol{u}_{4}\right)^{2}+\boldsymbol{u}_{3}^{2}} \text {. }
$$

We are done with the first entries of the first rows. Now we proceed with the second entries there:

$$
u_{2}+i u_{1}=\frac{-2 e z}{1+2 m-2 i x_{1}}
$$

We write $x_{2}, x_{3}$ in polar coordinates as follows:

$$
\begin{aligned}
& \quad x_{2}=r \sin \theta, x_{3}=r \cos \theta . \\
& \text { Recall } e=\cos x_{4}+i \sin x_{4} . \text { Let } \\
& N=(2 m+1)^{2}+4 x_{1}^{2} .
\end{aligned}
$$

Then (7) is equivalent to the system:

$$
\begin{gathered}
N u_{1}=-2 r\left[(2 m+1) \cos \left(x_{4}-\theta\right)-2 x_{1} \sin \left(x_{4}-\theta\right)\right], \\
N u_{2}=2 r\left[(2 m+1) \sin \left(x_{4}-\theta\right)+2 x_{1} \cos \left(x_{4}-\theta\right)\right]
\end{gathered}
$$

Since $r, m, x_{1}$ are already determined (in terms of the element $Z$ ), the values of both $\cos \left(x_{4}-\theta\right), \sin \left(x_{4}-\theta\right)$ are uniquely determined.

The equality of the first entries in second rows of (6) is equivalent to:

$\sin \left(x_{4}+\theta\right)-i \cos \left(x_{4}+\theta\right)=d\left(i u_{1}-u_{2}\right) / 2 r u$.

Since the right hand side is a complex number of length one, the left hand side is uniquely determined (in terms of the matrix Z)
We have thus determined all four parameters of (5) in terms of the matrix $Z$. The equality of the second entries in second rows of (6) is satisfied, too.

Overall, we have shown that all elements of $U(2)$, which cannot be represented by (5), form the circle

$$
\left[\begin{array}{ll}
1 & 0 \\
0 & d
\end{array}\right]
$$


This is the case where $u_{4}=1$ (which implies $u_{1}=u_{2}=u_{3}=0$, and subsequently $x_{-1}=m=0$ ), and (6) does not hold. Regarding the stationary subgroup: based on (5) simple calculation finishes the proof of Theorem 2.

Remark 3. Since this stationary subgroup is not an invariant one in $L_{G}$, the oscillator group (4) does not induce a group operation on its homogeneous space $L$. Compare it with a different outcome in the $U(1,1)$ case (see Remark 2 of Section III). The content of the next section is reproduced from [6].

\section{EMBEDDING OF U $(1,1)$ INTO U(2) AND GENERALIZATIONS TO HIGHER DIMENSIONS: THE SVIDERSKIY FORMULA}

Let us start with a brief discussion of the general case. Formula

$$
n=s m
$$

sets up a linear bijection between vector spaces of Lie algebras $u(p, q)$ and $u(p+q)$ : (9) is mentioned on p. 219 of [7]. Here $s$ is a diagonal matrix with $p$ ones and $q$ negative ones on the principal diagonal and $u(p, q)$ is the set of all $p+q$ by $p+q$ matrices $m$ which satisfy our (2) above, given nonnegative integers $p$ and $q$. Obviously,

$$
m=s n
$$

is the formula for the inverse mapping from $u(p+q)$ onto $u(p, q)$.

Formulas (9 and 10) might be viewed as giving canonical linear correspondence between $u(p, q)$ and $u(p+q)$ but how about correspondence between Lie groups $U(p, q)$ and $U(p+q)$ ?

The research in this direction has been started (see [8]) by the second author together with late Oleg $\mathrm{S}$. Sviderskiy (31 July 1969 - 30 March 2011). As a tribute to Oleg, it is now suggested that the formula for the canonical correspondence between groups $U(p, q)$ and $U(p+q)$ be known as the Sviderskiy formula; it is presented below as Theorem 3.

We first describe how $U(1,1)$ sits in $U(2)$. This is defined by the following function $\boldsymbol{h}$ from $D=U(2)$ : the image of a matrix $Z=\left[\begin{array}{ll}z_{1} & z_{2} \\ z_{3} & z_{4}\end{array}\right]$ from $U(2)$ is the matrix $V$ with entries

$$
v_{1}=d / z_{4}, v_{2}=z_{2} / z_{4}, v_{3}=-z_{3} / z_{4}, v_{4}=1 / z_{4} \text {; }
$$

here $d$ is the determinant of $Z$. Notice that the determinant of $V$ equals $z_{1} / z_{4}$.

Proposition 2. The mapping (10) is only undefined for elements $Z$ on the torus $z_{1}=$ $z_{4}=0$ in $D=U(2)$. The image is the entire $F=U(1,1)$. In terms of Lorentzian metrics (introduced in [2] on both $D$ and $F$ ) the mapping (10) is conformal. The tangent mapping (or the differential of $\boldsymbol{h}$ ) at the neutral element of $D$ is exactly our (10).

Here we only notice that correspondence (10) is similar to the one established in [2] (Theorem 6) whereas other details of the proof are to be presented elsewhere.

Remark 1. Significant part of what is discussed in this section, also makes sense in the $S O(p, q)$ vs $S O(p+q)$ context.

Remark 2. Having in mind the realization (4) of the oscillator Lie group, the following seems to be one of the most natural ways to introduce $F_{G}$, a subgroup of $G=S U(2,2)$ locally isomorphic to $U(1,1)$. In the context of [2] (Section 6), the blocks of a generic element in $F_{G}$ are as follows: $\mathrm{B}=\mathrm{C}=0, \mathrm{~A}=Z$, $\mathrm{D}=q$ where $Z$ is from $U(1,1)$ and $q^{2}$ times determinant of $Z$ is one. If to proceed similarly to how we did in the proof of the above Theorem 2, then the homogeneous space $F$ inherits the group operation from $F_{G}$ since the stability subgroup is central in $F_{G}$. Namely, this stability subgroup consists of scalar matrices $i,-i, 1,-1$ and $F$ is isomorphic to $U(1,1)$.

Proposition 3 below relates the three worlds together in a more specific way than it has been done in [9] and in [2] (Theorem 6). In that Proposition 3 we view $F=U(1,1)$ as a subset of $D$ defined by (3.2), whereas we choose another $L$ rather than the one described in Theorem 2 and Remark 3 of Section II.

Proposition 3. $F<L<D$, embeddings of manifolds.

Proof. Let us conjugate the oscillator subgroup (4) in $S U(2,2)$ in such a way that the resulting orbit of a certain matrix $X$ has the following property: it contains all elements of $U(2)$ with non-zero entry $Z_{4}$. In other words, the analogue of the circle (2.8) for this orbit will be contained in the torus $z_{1}=z_{4}=0$. It is easy to verify that the following element $g_{0}$ in $S U(2,2)$ takes the matrix 


$$
X=\left[\begin{array}{cc}
0 & -1 \\
-1 & 0
\end{array}\right]
$$

into negative one: blocks B and C of $g_{0}$ both vanish, whereas

$$
\mathrm{A}=\left[\begin{array}{ll}
0 & i \\
i & 0
\end{array}\right], \quad \mathrm{D}=\left[\begin{array}{ll}
i & 0 \\
0 & i
\end{array}\right]
$$

The inverse of $g_{0}$ is its own negative, from where it follows that $-g_{0} L_{G} g_{0}$ is an appropriate subgroup conjugate to the group $L_{G}$ defined by (4). The corresponding circle (points of which do not belong to the orbit) is the image of (8) under $g_{0}$ :

$$
\left[\begin{array}{ll}
0 & d \\
1 & 0
\end{array}\right]
$$

Clearly, this circle is on the torus (points of that torus do not belong to $F$ ). Proposition 3 is proved.

We now proceed with the Sviderskiy formula which defines an embedding of $U(p, q)$ into $U(p+q)$ as manifolds. This mapping is defined as a fractional linear application of a certain $2 n$ by $2 n$ matrix $W$ to (all) matrices in $U(p, q)$; here $n=p+q$. The $n$ by $n$ blocks $A, B, C, D$ of the matrix $W$ are defined as follows:

$$
\begin{aligned}
& \mathrm{A}=\mathrm{D}=\left[\begin{array}{ll}
I_{p} & 0 \\
0 & 0
\end{array}\right] \\
& \mathrm{B}=\mathrm{C}=\left[\begin{array}{ll}
0 & 0 \\
0 & I_{q}
\end{array}\right]
\end{aligned}
$$

where $I_{p}$ (respectively, $I_{q}$ ) stand for the unit matrix of size $p$ (respectively, of size $q$ ).

Theorem 3 (the Sviderskiy formula). The fractional linear application of the above introduced matrix $W$ is defined for all matrices in $U(p, q)$, and $U(p, q)$ is in a oneto-one correspondence with its image. The inverse mapping is also defined as the fractional linear transformation (by the same matrix $W$ ).

The proof is to be presented elsewhere.

Remark 3. The above (10) is a special case of the Sviderskiy formula.

\section{ACKNOWLEDGMENTS}

As regards the first author, this project was funded [in part] by BU UROP.

\section{REFERENCES}

1. Segal I, D. Vogan, Z. Zhou 1995 Chronometric Particle Theory I. An Alternative to the Higgs Mechanism (with D. Vogan and Z. Zhou), Unpublished (available from http://dedekind.mit.edu/segalarchive/).

2. A V Levichev 2011 Pseudo-Hermitian realization of the Minkowski world through the DLF-theory Physica Scripta N.1 1-9.

3. Paneitz S M and I. E. Segal 1982 Analysis in space-time bundles I: General considerations and the scalar bundle Journal of Functional Analysis 47 78-142.

4. A V Levichev 2009 Oscillator Lie algebra and algebras $u(2), u(1,1)$, as a single matrix system in $\mathrm{u}(2,1)$. In: "Lie algebras, algebraic groups, and the theory of invariants"/Proceedings of the Summer School-Conference, Samara, Russia, June 8-15 2009, 3234.

5. J Hilgert, K.-H. Hofmann, and J D Lawson, Convex Cones and Semigroups. Clarendon Press, 1989.

6. A $\vee$ Levichev 2011 On embeddings of $U(p, q)$ into $U(p+q)$ : the Sviderskiy formula, in preparation.

7. B A Dubrovin, A T Fomenko, S P Novikov, Modern Geometry - Methods and Applications: Part I: The Geometry of Surfaces, Transformation Groups, and Fields. Springer, 1991.

8. A V Levichev and O S Sviderskiy 2009 Lie groups $U(p, q)$ of matrices as a single system based on fractional linear transformations: I. General consideration and cases $p+q=2,3$. In:Proceedings of the International Conference "Contemporary Problems of Analysis and Geometry проблемы анализа и геометрии" Sobolev Institute of Mathematics of the Siberian Division of the Russian Academy of Sciences, Novosibirsk 6869.

9. A V Levichev 2003 Three symmetric worlds instead of the Minkowski space-time, Trans.RANS, Ser. MMM\&C, 7 n.3-4 87-93 


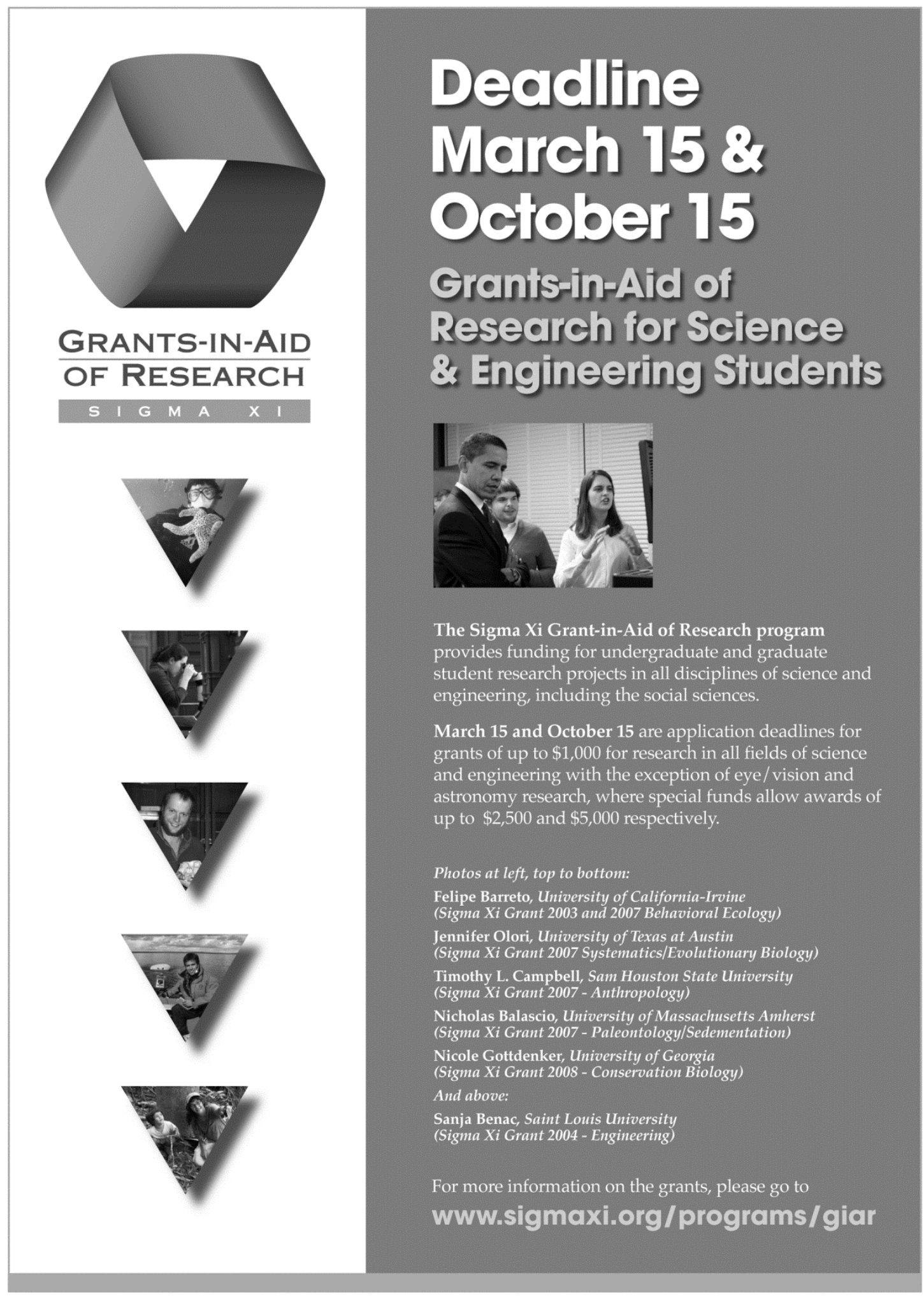

